\title{
MODELO DE DESARROLLO DE COMERCIO ELECTRÓNICO B2C EN LOS PAÍSES MENOS DESARROLLADOS: El caso peruano*
}

\author{
Antonio Díaz Andrade \\ PROFESOR DE TECNOLOGÍAS DE INFORMACIÓN, ESAN
}

\section{Resumen}

Esta investigación propone un modelo de desarrollo para el comercio electrónico de empresa a consumidor (B2C) en los países menos desarrollados, a partir de la situación del comercio electrónico en el Perú. Para hallar características comunes que permitan definir el modelo, se realizan entrevistas semiestructuradas a tres sectores de profesionales vinculados al desarrollo del comercio electrónico en el Perú: los responsables de la definición de políticas y normas sobre comercio electrónico, los proveedores de servicios de Internet (ISP), y los gerentes y directores de empresas inmersas en las actividades de comercio electrónico. Se propone a priori que el modelo contemple la situación de la infraestructura tecnológica nacional, la difusión de los medios de pago, la situación de la distribución física y la cultura de compra en línea en el Perú.

\section{A} pesar de la abrupta caída del índice compuesto de NASDAQ en abril del año 2000, arrastrado por el colapso de las hasta ese entonces promisorias empresas puntocom, Unctad pronostica que la tecnología de información y comunicaciones (TIC), especialmente Internet, continuará guiando el crecimiento económico del

Ponencia presentada en la $15^{\text {th }}$ Information Resources Management Association (IRMA) International Conference, realizada en Filadelfia, Estados Unidos, en mayo de 2003. mundo (World Trade 2002). Es de esperar, por tanto, que esta tecnología y el comercio electrónico sean los medios apropiados para incrementar la competitividad de las empresas y de los países, con la finalidad de mejorar la calidad de vida de las poblaciones (Unctad 2002); aproximadamente el 30\% del total del crecimiento económico en Estados Unidos se atribuye a esta tecnología (Schlögl 2001).

Sin embargo, el dispar crecimiento de Internet, en particular, y de la tecnología 
de información y comunicaciones, en general, entre los diferentes grupos sociales en el mundo durante la última década ha originado el problema de la brecha digital (Norris 2001), que se convierte en una amenaza para los países menos desarrollados.

\section{Objetivo y metodología}

Frente a este panorama, el objetivo de esta investigación es proponer un modelo de desarrollo para el comercio electrónico de empresa a consumidor (B2C) en los países menos desarrollados, a partir de la situación del comercio electrónico en el Perú.

Con el propósito de recolectar información relevante, se hizo una revisión bibliográfica de la literatura hasta hoy existente sobre comercio electrónico en los países menos desarrollados. Luego, se aplicó entrevistas semiestructuradas, de manera diferenciada, a tres sectores de profesionales vinculados al desarrollo del comercio electrónico en el Perú: los responsables de la definición de políticas y normas sobre comercio electrónico, los proveedores de servicios de Internet (ISP), y los gerentes y directores de empresas inmersas en las actividades de comercio electrónico.

Los resultados obtenidos fueron analizados a fin de encontrar características comunes que permitieran definir un modelo de desarrollo de comercio electrónico en países en desarrollo. Se propuso $a$ priori que el modelo contemple la situación de la infraestructura tecnológica nacional, la difusión de los medios de pago, la situación de la distribución física y la cultura de compra en línea en el Perú.

\section{Marco conceptual}

La creación de Internet en 1969, como una red de uso militar y académico, y su posterior uso comercial en 1991 trajeron como consecuencia una imprevista popularidad del uso del correo electrónico. Después, en 1993, la aparición de software para navegar en la web originó un amplio rango de capacidades de transporte en Internet, y se pudo enviar fotos, texto, video y audio (Westland y Clarke, 1999). Más tarde, se iniciaron las primeras transacciones a través de la web.

El comercio electrónico se define como «la compra y venta de bienes sobre medios digitales, específicamente [...] la web para permitir el remodelamiento de los negocios, mercados y la ventaja competitiva» (Kalakota y Robinson, 1999: 23).

Tres grandes fuerzas explican el desarrollo del comercio electrónico: la digitalización, entendida como mayor potencia de los computadores y mayores anchos de banda disponibles a precios cada vez menores; la globalización, por la que cada vez el mundo es más pequeño en términos de distancias y más grande en términos de tamaño del mercado; y la desregulación, estimulada por la percepción de que el libre mercado es el mejor distribuidor de los recursos (Arroyo, Herrera, Temoche, Vilches y Whittembury, 2001). Estas tres fuerzas pueden cambiar la cadena de valor de cualquier empresa sin importar el tamaño de ésta.

Así, una empresa electrónica (e-business) es aquella que utiliza tecnología de información, especialmente de redes en tiempo real, para el desarrollo de sus actividades. Se podría considerar como empresas electrónicas no solamente a aque- 
llas que venden productos en línea, sino también a aquellas que utilizan tecnología de redes en sus procesos productivos, cadena de abastecimiento, actividades de marketing, automatización de ventas y servicio al cliente (Choi y Whinston, 2000).

La manera como las empresas utilicen Internet podría marcar la diferencia entre el éxito y el fracaso. Los orígenes de la ventaja competitiva se mantienen invariables, según Porter (2001); la integración de la tecnología de Internet en la estrategia empresarial permitirá fortalecer las fuentes de ventajas competitivas, pero en ningún caso podrá reemplazarlas. El comercio electrónico es, en definitiva, una forma de hacer negocios (Borenstein y Saloner, 2001), aunque modifica la forma como las empresas generan valor. Hoy «el tiempo de respuesta al mercado, la innovación y la calidad se han convertido en requisitos de supervivencia de las organizaciones» (Westland y Clarke, 1999: 53). Internet permite intercambiar y difundir datos hacia cualquier parte del mundo a un costo prácticamente despreciable, otorgándole a las empresas la oportunidad de formar alianzas y distribuir información sin las limitaciones que tradicionalmente ha impuesto la geografía (Lekse y OlivasLuján, 2001). De esta manera, si bien en el corto plazo las empresas de los países desarrollados podrían ser las más beneficiadas, en el largo plazo las empresas de los países menos desarrollados lograrían los mejores beneficios, dado que no tendrían que recorrer el largo camino de la curva de aprendizaje que debieron trazar las primeras (Panagariya 2000).

En cuanto a las repercusiones de este fenómeno, el desempeño que puedan alcanzar las empresas electrónicas se refle- jará en diferentes formas en las economías, ya sea a través de la recaudación de impuestos o la mejora del nivel de empleo; por tanto, sus consecuencias deben ser sopesadas. Algunos autores afirman que el comercio electrónico puede ayudar a mejorar sustancialmente la competitividad de un país (Sprano y Zakak, 2000); de ahí la importancia de que los estados realicen las acciones necesarias para superar las barreras que representan la educación deficiente de la población y la pobre infraestructura tecnológica, características comunes en los países menos desarrollados. El rol que les compete a los gobiernos es el de adecuar la normatividad legal no sólo para no obstruir, sino para promover el comercio electrónico, más aún teniendo en cuenta que en la mayoría de los países el Estado es el principal comprador.

Finalmente, los proveedores de servicios de Internet (ISP) tienen una gran responsabilidad en el desarrollo del comercio electrónico, pues los servicios que ofrecen -desde el uso de hardware y software hasta el acceso a redes- se vuelven indispensables para la existencia de empresas electrónicas.

\section{Comercio electrónico en el Perú}

Los empresarios de los países menos desarrollados enfrentan grandes dificultades tanto para crecer en sus mercados domésticos como para ingresar a los mercados internacionales. En este sentido, el número de empresas que desarrollan negocios en línea en el Perú es muy reducido; si bien el 96\% de las dos mil empresas más importantes están conectadas a Internet, solamente el $50 \%$ de ellas tienen página web, mientras que apenas el $18 \%$ practi- 
ca el comercio electrónico: $88 \%$ con operaciones $\mathrm{B} 2 \mathrm{~B}^{1}$ y $38 \%$ con operaciones B2C (Telefónica, 2002). Además, el 90\% de las transacciones minoristas en línea está concentrado en solamente cinco empresas $^{2}$ (Entrevista Edwin San Román, Osiptel, 2002).

Para desarrollar negocios electrónicos son indispensables tres elementos: el acceso a Internet, la disponibilidad de medios de pago y, tratándose de bienes tangibles, la distribución física de los productos (Mann 2000), todos ellos sustentados en una cultura de compras en línea. El modelo de desarrollo de los negocios electrónicos propuesto está representado en la siguiente figura.

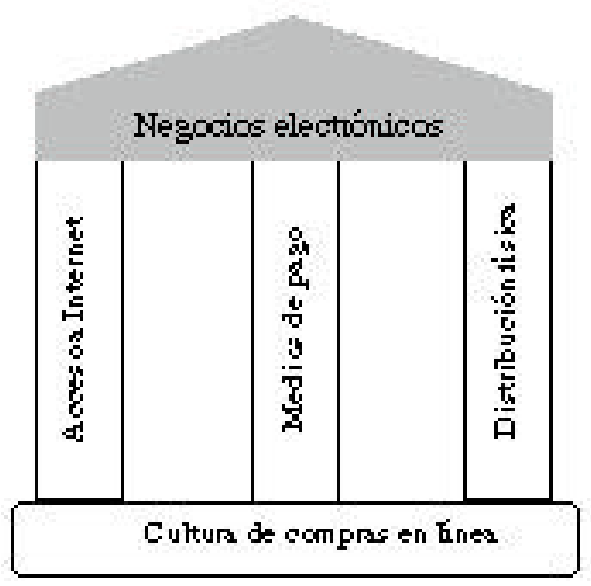

1. Los mayores montos negociados en línea bajo la modalidad B2B en el Perú corresponden a las transacciones realizadas entre empresas mineras y sus proveedoras.

2. El supermercado E. Wong (www.ewong.com), la tienda virtual y la agencia de viajes del informativo en línea peru.com (www.peru.com), la florería Rosatel (www.rosatel.com), la tienda por departamentos Saga-Falabella (www, sagafalabella.com.pe), y la tienda virtual del portal del diario El Comercio (www.ecstore.com).

\subsection{Acceso a Internet}

El Perú es un país con una pobre infraestructura de tecnología de información y comunicaciones, tal como se aprecia en el cuadro adjunto. Sin embargo, las cifras permiten inferir que, en comparación con otros países, la proporción de usuarios de Internet es muy alta frente al número de líneas de telefonía (fija o móvil), el número de computadoras personales o frente al número de hosts. Esto se explica por el fenómeno del acceso a Internet a través de las cabinas públicas, modelo que fue desarrollado a mediados de los noventa por la Red Científica Peruana. Así, de 580 cabinas públicas en diciembre de 1999, el Perú pasó a tener 1740 en junio de 2001 y la cifra sigue creciendo. Actualmente el Perú ocupa el puesto número 13 en una clasificación de países con mayor índice de acceso público a Internet (Harvard University, citado por Telefónica 2002).

\subsection{Medios de pago}

Los medios de pago en Internet siguen siendo insuficientes para lograr una expansión de los negocios electrónicos en el Perú. El nivel de 'bancarización' es muy reducido y el número de titulares de tarjetas de crédito es muy bajo con respecto a países más desarrollados. Hacia fines del año 2001, sólo el 24,96\% del volumen de los recursos del PBI eran administrados por los bancos peruanos (Asbanc, 2002), mientras que, hasta el año 2000, solamente el $9 \%$ de los hogares de Lima -la capital y a la vez la ciudad más importante y desarrollada del Perú- poseía una tarjeta de crédito (INEI, 2000). Algunos bancos locales desarrollaron tarjetas de compras exclusivas para Internet, tales como la tarjeta viaBCP del Banco de Crédito del Perú 
Cuadro 1

Principales indicadores TIC (por cada 100 habitantes)

\begin{tabular}{|l|r|r|r|r|r|}
\hline Países & $\begin{array}{c}\text { Telefonía } \\
\text { fija }\end{array}$ & $\begin{array}{c}\text { Telefonía } \\
\text { móvil }\end{array}$ & $\begin{array}{c}\text { Computadoras } \\
\text { personales }\end{array}$ & $\begin{array}{c}\text { Usuarios } \\
\text { Internet }\end{array}$ & $\begin{array}{c}\text { Hosts } \\
\text { (c. 1 000 hab.) }\end{array}$ \\
\hline Estados Unidos & 66,5 & 44,4 & 62,3 & 34,6 & 292,8 \\
Unión Europea & 57,5 & 60,5 & 24,4 & 38,0 & 75,0 \\
Países Escandinavos & $65,0+$ & $66,0+$ & $60,0+$ & $50,0+$ & 100,0 \\
Argentina & 21,6 & 18,6 & 5,3 & 5,3 & 12,4 \\
Brasil & 21,8 & 16,7 & 6,3 & 3,5 & 9,5 \\
Chile & 23,9 & 34,0 & 8,4 & 11,8 & 7,9 \\
Colombia & 17,1 & 7,6 & 4,2 & 1,7 & 1,3 \\
México & 13,7 & 21,7 & 6,9 & 2,3 & 9,1 \\
Perú & 7,6 & 5,9 & 4,8 & 12,0 & 0,5 \\
Uruguay & 28,3 & 15,5 & 11,0 & 11,1 & 21,1 \\
Venezuela & 11,2 & 26,4 & 5,3 & 5,0 & 0,9 \\
\hline
\end{tabular}

Fuentes: International Telecommunications Union (ITU), Central Intelligence Agency (CIA),CyberAtlas, INEI y Osiptel.

(www.viabcp.com) y la tarjeta Netactiva24 de Interbank (www.interbank.com.pe), ambas asociadas a una cuenta de ahorros. El Banco Wiese Sudameris (www.wiese. com.pe) lanzó la tarjeta Pagum MasterCard, caracterizada porque no necesariamente tiene que estar asociada a cuenta alguna en el banco. De ellas, la tarjeta via$\mathrm{BCP}$ es la que más aceptación tiene, quizás por el hecho de que el Banco de Crédito del Perú es el banco más grande del país y concentra el mayor número de clientes; ello aunado a una intensiva campaña de promoción. La tarjeta viaBCP tiene alrededor de 27 mil usuarios, que consumen aproximadamente 350 dólares mensuales; el $97 \%$ de dichas transacciones se realizan con establecimientos fuera del país.

Además del problema de la escasez de medios de pago, se presenta el de la diversidad de pasarelas de pago, ya que muchas de las tarjetas de crédito bancarias y aquellas emitidas por establecimien- tos comerciales han desarrollado una infraestructura de pago propietaria, lo cual complica la operación. Algunos negocios le permiten al comprador pagar en efectivo al recibir la mercadería solicitada; sin embargo, esta opción obliga a realizar verificaciones previas para evitar el rechazo de la transacción y los consiguientes gastos de envío por mercadería devuelta.

\subsection{Distribución física}

Cuando por tratarse de bienes tangibles se hace necesaria la distribución física, las empresas en línea peruanas han optado por subcontratar este servicio. Las tarifas por entrega se establecen, principalmente, en función de la distancia entre el centro de distribución y el lugar indicado por el comprador; por ello, y considerando que la mayoría de las empresas electrónicas están ubicadas en Lima, son pocos los casos de entregas en el interior del país, ya que los cargos por distribución se elevarían significativamente. Algunas empre- 
sas han iniciado una expansión abriendo tiendas en otras ciudades, lo cual simplifica la distribución física de sus pedidos mediante la integración de las operaciones on-line y off-line.

En cuanto al exterior, se ha avanzado muy poco en términos de recurrir al comercio electrónico como canal para las exportaciones. Al respecto, debería evaluarse detenidamente cuál sería la oferta exportable de productos peruanos susceptible de ser vendida a través de Internet y bajo qué modelo de negocio podría hacerse la operación. Algunas empresas electrónicas peruanas han desarrollado con bastante éxito ventas en el exterior para productos que son distribuidos localmente (Santana y Díaz, 2002).

\subsection{Cultura de compras en línea}

A diferencia de países más desarrollados, en el Perú el hábito de compras por catálogo no está difundido, razón por la cual las ofertas comerciales a través de Internet enfrentaron a un público no familiarizado con escoger y ordenar de manera remota, sin tener la mercadería al alcance de sus manos. Cuando se les preguntó a los usuarios de Internet en Lima para qué utilizaban este medio, ellos señalaron espontáneamente una serie de acciones, que iban desde el correo electrónico hasta la descarga de videos, pero no mencionaron la compra como una práctica frecuente (Telefónica, 2002).

En un estudio realizado para definir los perfiles de los usuarios de Internet en Lima, se encontró que solamente el $4 \%$ de los usuarios que acceden a través de cabinas públicas (más del $70 \%$ del total) han comprado alguna vez en Internet, mientras que el $15 \%$ de aquellos que acceden desde el hogar (9\% del total) y el $13 \%$ de aquellos que acceden desde el centro de trabajo o de estudios (17\% del total) lo han hecho alguna vez. Estas cifras indican que el $92 \%$ de usuarios nunca han realizado transacción alguna por Internet. Entre estos usuarios que no han comprado por Internet, $18 \%$ aduce falta de confianza y $38 \%$ considera inseguro este canal (Apoyo, citado en Telefónica 2002).

Es responsabilidad del gobierno crear el clima de confianza apropiado para permitir que los agentes económicos desarrollen negocios en línea. Para ello es necesario un marco legal que proteja la seguridad de las transacciones (Goldstein y O'Connor, 2001). En este sentido, el sistema jurídico peruano cuenta con una completa normatividad legal, tal como se muestra en el anexo. Si bien son de reciente dación, algunos expertos opinan que en el Perú las leyes han avanzado más rápido que las prácticas comerciales en línea.

\section{Discusión}

Tal como lo señalaran Kirkman y Sachs (2001), para aprovechar efectivamente las ventajas que proporciona la tecnología de información, y dentro de ella el comercio electrónico, se requiere una decidida voluntad política del Estado y, a la vez, un adecuado liderazgo empresarial.

En el caso peruano, las normas emitidas para la privatización de los servicios de telecomunicaciones, la promoción de estos servicios en zonas rurales, el proyecto de uso de tecnología de información aplicada a la educación a través del Programa Huascarán(www.huascaran.gob.pe), el proyecto de gobierno electrónico, los foros sobre la sociedad de la información 
que se están organizando y las normas que buscan promover el uso de Internet como un canal comercial son muestras de la voluntad política del Estado para promover la sociedad de la información. De hecho, el comercio electrónico es una característica importante de la sociedad de la información.

Por otro lado, una serie de acciones reflejan el ímpetu de la iniciativa privada para convertirse en actor importante en el mundo de la sociedad de la información: las empresas tradicionales peruanas que ingresaron al mundo de Internet, las ideas de negocios en línea hoy operando, la banca electrónica y el lanzamiento de las tarjetas de compras en Internet, la propuesta del Plan Puyhuán para definir un modelo de desarrollo rural sostenible mediante el uso de tecnología de información (www.setinedic.edu.pe/proyectoPuyhuan/ modeloplan.htm) y el creciente número de cabinas públicas de Internet

Si bien el ciudadano peruano ha mostrado interés en acceder a la tecnología de información, aún no está convencido de utilizar Internet como medio de transacción. Se espera que las iniciativas mencionadas en los párrafos anteriores causen un efecto multiplicador que contribuya a alentar la realización de transacciones de manera cómoda y segura desde una computadora.

Dadas las condiciones particulares existentes en un país poco desarrollado como el Perú, con manifiestas desigualdades económicas y una notoria brecha digital al interior de su sociedad, es poco probable que tenga éxito un modelo de comercio electrónico similar al de los países más desarrollados. En éstos el acceso doméstico a Internet es muy común, los medios de pago en Internet están ampliamente difundidos, se dispone de un adecuado servicio de distribución física y se trabaja sobre una tradición de compras por catálogo.

En el Perú, la extensa y creciente red de cabinas públicas debería aprovecharse no solamente como medio de acceso a Internet, sino como centro de pago para las órdenes realizadas en línea. Al respecto, ya figura dentro de los planes del proyecto de gobierno electrónico convertir a los administradores de las cabinas públicas en responsables de cobranza por los trámites que se pudieran ofrecer en línea; lo cual tendría un efecto reflejo en el comercio electrónico. De esta manera, las empresas podrían establecer acuerdos con algunas de las cabinas públicas a fin de convertirlas en centros autorizados de cobranza para los productos y servicios que comercializan en línea. Así, se beneficiarían tanto los dueños de cabinas públicas - dada la intensa competencia que impide generar excedentes suficientes para hacer viable el negocio en el largo plazo- como las empresas que comercializan a través de Internet, que podrían ofrecer un canal adicional a sus clientes $\mathrm{y}$, al mismo tiempo, conquistar nuevos mercados.

Bajo este esquema de negocios en línea, los operadores logísticos incrementarían su volumen de operaciones, actualmente pequeño, lo que a su vez podría reducir los costos de distribución. De este modo, los proveedores de servicios de Internet deberían estar preparados para atender la necesidad de mejores características de conexión; asimismo, les resultaría atractivo ampliar su red de servicios para alcanzar una mayor cobertura geográfica en aquellas zonas donde se perciba una oportunidad de mercado. 


\section{Referencias bibliográficas}

ARROYO, J. A.; HERRERA, G. A.; TEMOCHE, L. F.; VILCHES, F. J. y WHITTEMBURY, J. T. 2001. Transición de las empresas peruanas hacia el comercio electrónico. Lima: ESAN. 2 vol. Tesis para optar el grado de Magíster en Administración.

\section{ASOCIACIÓN DE BANCOS DEL PERÚ}

(ASBANC). 2002. http://www.elcomercioperu.com.pe/Texto/Html/2002-09-26/ Econom4683.html (5 de oct. de 2002).

BORENSTEIN, S. y SALONER, G. 2001. Economics and Electronic Commerce. Journal of Economic Perspectives. Vol. 15, n. ${ }^{\circ}$, págs. 3-12.

CHOI, S. Y. y WHINSTON, A. B. 2000. The Internet Economy: Technology and Practice. Austin, TX: SmartEcon Publishing.

GOLDSTEIN, A. y O'CONNOR, D. 2001. Navigating between Scylla and Charybdis. OECD Observer. January, n. ${ }^{\circ} 224$, págs. 72-74.

PERÚ. INSTITUTO NACIONAL DE ESTADÍSTICA E INFORMÁTICA. 2000. Tecnologías de información y comunicaciones en los hogares en Lima metropolitana. Lima: INEI.

KALAKOTA, R. y ROBINSON, M. 1999. EBusiness Roadmap for Success. Reading: Addison-Wesley.

KIRKMAN, G. y SACHS, J. 2001. Subtract the Divide. World Link. January-February, págs. 60-65.
LEKSE, W. J. y OLIVAS-LUJÁN, M. R. 2001. Getting Firms in Developing Countries on the E-Commerce Highway. International Journal of e-Business Strategy Management. August-September, vol. 3, n. ${ }^{\circ} 1$, págs. 45-53.

MANN, C. L. 2000. Electronic Commerce in Developing Countries: Issues for Domestic Policy and WTO Negotiations. http:// www.iie.com/CATALOG/WP/2000/003.pdf (23 de jul. de 2002).

NORRIS, P. 2001. Digital Divide? Civic Engagement, Information Poverty, and the Internet Worldwide. Cambridge: Cambridge University Press.

PANAGARIYA, A. 2000. E-Commerce, WTO and Developing Countries. World Economy. Vol. 23, n. ${ }^{\circ}$ 8, págs. 959-978.

PORTER, M. E. 2001. Strategy and the Internet. Harvard Business Review. March, vol. 9, n. ${ }^{\circ}$, págs. 63-78.

SANTANA, M. y DÍAZ, A. 2002. Inca Foods: Reaching New Customers Worldwide. En M. Raisinghani (Ed.), Cases on Worldwide e-Commerce: Theory in Action. Hershey, PA: Idea Group Publishing.

SCHLÖGL, H. 2001. Digital Lessons for Digital Policies. OECD Observer. January, n. ${ }^{\circ} 224$, págs. 41-42.

SPRANO, E. y ZAKAK, A. 2000. E-Commerce Capable: Competitive Advantage for Countries in the New World E-Conomy. Competitiveness Review. Vol. 10, n. ${ }^{\circ}$ 2, págs. 114-122. 
TELEFÓNICA DEL PERÚ. 2002. La sociedad de la información en el Perú: presente y perspectivas 2003-2005. Lima: Servicios Editoriales del Perú.

UNITED NATIONS CONFERENCE ON TRADE AND DEVELOPMENT. 2002. E-Commerce and Development Report. Internet version prepared by UNCTAD Secretariat. http://r0.unctad.org/ecommerce/docs/edr02_en/ecdr02.pdf (4 de dic. de 2002).
WESTLAND, J. C. y CLARKE, T. H. K. 1999. Global Electronic Commerce: Theory and Cases. Cambridge: The MIT Press.

WORLD TRADE. 2002. E-Commerce Yet to Improve Developing World. World Trade. April, vol. 15, n. ${ }^{\circ}$, pág. 14. 


\section{Anexo:}

Normas legales relativas a telecomunicaciones, tecnología de información y comercio electrónico

\begin{tabular}{|c|c|}
\hline${\text { Decreto Legislativo N. }{ }^{\circ} 681(11 / 10 / 91)}$ & Regula el archivo de documentos digitales. \\
\hline${\text { Decreto Legislativo N. }{ }^{\circ} 702(05 / 11 / 91)}$ & $\begin{array}{l}\text { Promueve la inversión privada en telecomuni- } \\
\text { caciones. }\end{array}$ \\
\hline Decreto Supremo N. ${ }^{\circ} 013-93-\mathrm{TCC}(28 / 04 / 93)$ & $\begin{array}{l}\text { Aprueba el texto de la Ley de Telecomunica- } \\
\text { ciones. }\end{array}$ \\
\hline Ley N. ${ }^{\circ} 26285(12 / 01 / 94)$ & $\begin{array}{l}\text { Dispone la desmonopolización progresiva de } \\
\text { los servicios públicos de telecomunicaciones. }\end{array}$ \\
\hline Decreto Supremo N. $.^{\circ} 011-94-\mathrm{TCC}(13 / 03 / 94)$ & $\begin{array}{l}\text { Aprueba el contrato de concesión entre el estado } \\
\text { peruano y las empresas Entel-Perú y CPTSA. }\end{array}$ \\
\hline $\begin{array}{l}\text { Resolución Ministerial N. }^{\circ} \text { 250-97-MTC } \\
(17 / 06 / 97)\end{array}$ & $\begin{array}{l}\text { Aprueba el Plan Nacional de Atribución de } \\
\text { Frecuencias. }\end{array}$ \\
\hline Decreto Supremo N. ${ }^{\circ} 020-98-\mathrm{MTC}$ & $\begin{array}{l}\text { Define los lineamientos de políticas de apertu- } \\
\text { ra del mercado de telecomunicaciones. }\end{array}$ \\
\hline $\begin{array}{l}\text { Lineamientos de libre y leal competencia } \\
(08 / 02 / 2000)\end{array}$ & $\begin{array}{l}\text { Osiptel señala como prohibidos algunos casos } \\
\text { de abuso de posición de dominio y prácticas } \\
\text { restrictivas. }\end{array}$ \\
\hline Regulación de la interconexión & Osiptel regula la interconexión entre empresas. \\
\hline Ley N. $^{\circ} 27291(24 / 06 / 2000)$ & $\begin{array}{l}\text { Permite la utilización de medios electrónicos } \\
\text { para la comunicación de la manifestación de } \\
\text { voluntad. }\end{array}$ \\
\hline Ley N. ${ }^{\circ} 27309(17 / 07 / 2000)$ & Incorpora delitos informáticos al Código Penal. \\
\hline Ley N. ${ }^{\circ} 27419(07 / 02 / 2001)$ & Reconoce la notificación por correo electrónico. \\
\hline Ley N. $^{\circ} 27269(28 / 05 / 2000)$ & $\begin{array}{l}\text { Crea infraestructura de firmas y certificados di- } \\
\text { gitales. }\end{array}$ \\
\hline $\begin{array}{l}\text { Resolución de Consejo Directivo } \\
\text { N. }{ }^{\circ} 015-2001-C D / O s i p t e l\end{array}$ & $\begin{array}{l}\text { Establece las condiciones de uso de los servi- } \\
\text { cios públicos de Internet. }\end{array}$ \\
\hline Resolución Suprema N. ${ }^{\circ}$ 292-2001-RE & $\begin{array}{l}\text { Crea la comisión multisectorial encargada de } \\
\text { definir las políticas y lineamientos para la ad- } \\
\text { ministración del nombre de dominio (ccTLD) } \\
\text { correspondiente al Perú y los criterios para la } \\
\text { delegación de la actividad de registro. }\end{array}$ \\
\hline Decreto Supremo N. ${ }^{\circ}$ 66-2001-PCM & $\begin{array}{l}\text { Define lineamientos para la masificación de } \\
\text { Internet. }\end{array}$ \\
\hline Resolución Ministerial N. ${ }^{\circ}$ 266-2002-PCM & Crea la Jefatura de Gobierno Electrónico. \\
\hline 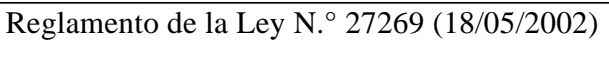 & $\begin{array}{l}\text { Reglamenta la ley de firmas y certificados di- } \\
\text { gitales. }\end{array}$ \\
\hline Decreto Supremo N. ${ }^{\circ}$ 67-2001-ED & Inicia el Plan Huascarán. \\
\hline Decreto de Urgencia N. ${ }^{\circ}$ 67-2001 (17/06/2001) & $\begin{array}{l}\text { Crea el Fondo Nacional para el Uso de Nuevas } \\
\text { Tecnologías de la Información (Fondunet). }\end{array}$ \\
\hline Ley N. $27806(02 / 08 / 2002)$ & Transparencia y acceso a la información pública. \\
\hline
\end{tabular}

Fuentes: Diversas. 


\section{Agradecimientos}

El autor desea expresar su reconocimiento a las personas que, con su valioso aporte, contribuyeron de manera desinteresada al desarrollo de este trabajo:

Miguel Aréstegui Matutti

Jefe del Centro de Documentación e Información Científica y Tecnológica

Consejo Nacional de Ciencia y Tecnología (Concytec)

\section{Pablo Bermúdez Mogni}

Jefe del Proyecto Gobierno Electrónico de la Secretaría de Gestión Pública

Presidencia del Consejo de Ministros

Mario Cámara Figueroa

Director Técnico de Normatividad y

Promoción

Instituto Nacional de Estadística e Informática (INEI)

Leonardo Carbonell Romero Director Ejecutivo de Políticas y Normas Instituto Nacional de Estadística e Informática (INEI)

José Antonio de Izcue A

Director gerente, Digital Way

Ronald Espinoza Icaza

Gerente general, Qnet

Sandro Marcone

Consultor de la Red Científica Peruana y ex director ejecutivo del Programa Huascarán

\section{Martín Moscoso Villacorta}

Jefe de la Oficina de Derechos de Autor Instituto Nacional de Defensa de la Competencia y de la Protección Intelectual (Indecopi)

\section{Jorge Muñiz Ziches}

Abogado del Estudio Muñiz, Forsyth, Ramírez, Pérez-Taiman y Luna-Victoria y ex congresista de la República; autor de la ley de firmas y certificados digitales

\section{Juan Carlos Olivera}

Jefe de Comercio Electrónico

www.elcomercioperu.com.pe

Javier Pardo Barber

Director gerente

www.rosatel.com

Juan Francisco Rosas Schwarz

Gerente general

www.peru.com

\section{Edwin San Román Zubizarreta}

Presidente

Organismo Supervisor de Inversión Privada en Telecomunicaciones (Osiptel)

Marco Suárez Lara

Adjunto a la Gerencia General

www.ewong.com

Luis Torres Valerín

Director de Investigación y Tecnología Instituto Nacional de Investigación y Capacitación de Telecomunicaciones (Inictel)

Rolando Toledo Vega

Gerente general

Red Científica Peruana 